\title{
Shear wave elastography diagnosis of the diffuse sclerosing variant of papillary thyroid carcinoma: A case report
}

\author{
NIANYU XUE ${ }^{1}$, YOUFENG XU ${ }^{1}$, PINTONG HUANG $^{2}$, SHENGMIN ZHANG $^{1}$, HONGWEI WANG $^{3}$ and FEI YU \\ ${ }^{1}$ Department of Ultrasonography, The Ningbo First Hospital, Ningbo, Zhejiang 315010; \\ ${ }^{2}$ Department of Ultrasonography, The Second Affiliated Hospital of Zhejiang University Medical College, Hangzhou, \\ Zhejiang 310000; ${ }^{3}$ Department of Pathology, The Ningbo First Hospital, Ningbo, Zhejiang 315010, P.R. China
}

Received January 22, 2016; Accepted May 25, 2016

DOI: $10.3892 /$ mco.2016.932

\begin{abstract}
The present study aimed to report the shear wave elastography (SWE) findings in a patient with the diffuse sclerosing variant of papillary thyroid carcinoma (DSVPTC). Since patients with DSVPTC may present with typical clinicopathological features and initially appear to have Hashimoto's thyroiditis, a thorough clinical evaluation and an early diagnosis are important. A 20-year-old female patient presented with a 1-month history of a neck mass and sore throat. Conventional ultrasound and SWE were performed using an AIXPLORER system with 14-5 MHz linear transducer. The patient had undergone total thyroidectomy and bilateral neck lymph node dissection, and an intraoperative pathology consultation to confirm the malignancy of lymph node metastasis. Pathological diagnosis was DSVPTC in both lobes, with lymph node metastases in the bilateral neck. The clinical presentation and serological findings were all indicative of Hashimoto's thyroiditis. Thyroid ultrasonography revealed diffuse enlargement of the both lobes, heterogenous echogenicity without mass formation, diffuse scattered microcalcifications and poor vascularization. SWE revealed stiff values of the thyroid: The mean stiffness was $99.7 \mathrm{kpa}$, the minimum stiffness was $59.1 \mathrm{kpa}$ and the maximum stiffness was $180.1 \mathrm{kpa}$. The maximum stiffness of the DSVPTC (180.1 kpa) was higher compared with the diagnostic criteria of malignant thyroid nodules $(65 \mathrm{kPa})$. SWE may be considered as a novel and valuable method to diagnose DSVPC.
\end{abstract}

\section{Introduction}

The diffuse sclerosing variant of papillary thyroid carcinoma (DSVPTC) was firstly reported by Vickery et al (1) in 1985.

Correspondence to: Dr Pintong Huang, Department of Ultrasonography, The Second Affiliated Hospital of Zhejiang University Medical College, 261 Qingchun Road, Hangzhou, Zhejiang 310000, P.R. China

E-mail: huangpintong@126.com

Key words: diffuse sclerosing variant of papillary carcinoma, ultrasonography, thyroid, elastography
The prevalence of this variant among all the patients with papillary thyroid carcinoma (PTC) ranged between 0.74 and 5.3\%, as reported in a larger series (2-4). This is characterized by high aggressiveness, a larger tumor size, a high incidence of cervical lymph node metastasis and distant metastasis (3,5-9). DSVPTC is also associated with frequent local recurrence and poor prognosis (8-11). Therefore, patients with DSVPTC must be treated by aggressive surgical intervention, including total thyroidectomy, central neck dissection, bilateral neck dissections and postoperative radioiodine treatment $(3,9,10,12)$. Patients with DSVPTC must be monitored closely during follow-up for any disease recurrence or metastasis $(3,7,9,12)$.

Clinically, the diagnosis of DSVPTC is often delayed by months or years as a result of the clinical similarities that it shares with autoimmune lymphocytic thyroiditis (also termed Hashimoto's thyroiditis) $(3,7,9,12)$. Due to the early spread of DSVPTC, early correct diagnosis and surgical treatment is crucially important (9). Currently, ultrasound (US) is generally considered to be the most accurate imaging modality for the evaluation and characterization of thyroid nodules (13). Since DSVPTC always permeates the entire gland causing diffuse thyroid enlargement without a dominant nodule, which is difficult to diagnose using US, it is more commonly diagnosed as Hashimoto's thyroiditis $(12,14,15)$. The present study was the first, to the best of our knowledge, to report shear wave elastography (SWE) diagnosis of DSVPTC of the thyroid.

\section{Patients and methods}

Patient. A 20-year-old female patient presented with a 1-month history of a neck mass and sore throat. The patient did not received non-steroidal anti-inflammatory drugs, chemotherapy, radiotherapy or immunotherapy. A physical examination revealed a diffuse palpable middle-neck mass protruding on both sides. The mass was tender, well-defined, firm and movable. Clinically, this was suggested to be thyroiditis. Serum concentrations of triiodothyronine (T3), thyroxine (T4), free triiodothyronine (FT3), free thyroxine (FT4), thyroidstimulating hormone (TSH), thyroglobulin antibody ( $\mathrm{Tg}-\mathrm{Ab}$ ), and thyroid peroxidase antibody (TPO-Ab) were measured using immunochemiluminescent assays and an automated analyzer (Unicel DXI800; Beckman Coulter, Inc., Brea, CA, 
$\mathbf{A}$
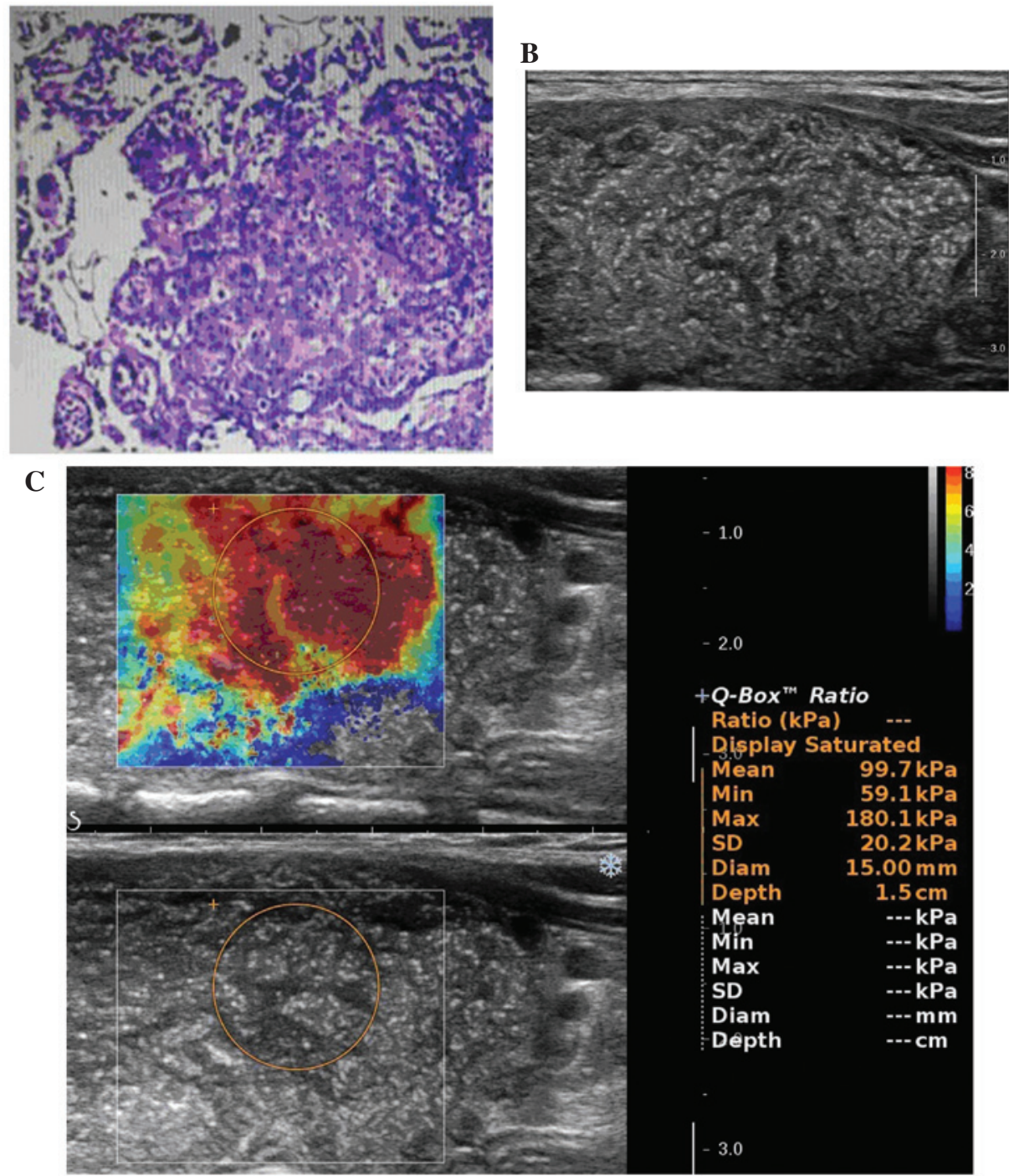

Figure 1. (A) The pathological diagnosis was diffuse sclerosing variant of papillary thyroid carcinoma. (B) Thyroid ultrasonography showed diffusely enlarged, heterogenous echogenicity without mass formation and diffuse scattered microcalcifications. (C) Shear wave elastography showed stiff values of the thyroid. The mean stiffness was $99.7 \mathrm{kpa}$, the minimum stiffness was $59.1 \mathrm{kpa}$ and the maximum stiffness was $180.1 \mathrm{kpa}$. SD, standard deviation; Diam, diameter.

USA). Conventional US and SWE were performed using an AIXPLORER system (Supersonic Imagine, Aix en Provence, France) with 14-5 MHz linear transducer. Written informed consent from the patient was obtained prior to SWE examination.

Conventional US and SWE. US examination was performed in transverse and longitudinal planes of the thyroid with the supine position. On conventional US, thyroid gland and lesion diameters, the extent of disease, echogenicity, microcalcifications, vascularization and cervical metastatic lymph nodes were assessed.

Following conventional US examination, SWE was performed during the US examination, using the same operator, US system and probe, which was placed on the neck with light constant pressure. The elastography image was overlaid on the B-Mode image. It provided a real-time map of elasticity. SWE provided results in a color box and region-of-interest (ROI), which can be positioned over the normal thyroid parenchyma and the nodule to measure the stiffness of each tissue. The SWE color box, in which blue and red areas corresponded to softer and stiffer regions, respectively, was moved to include the target lesion. When the cine loop was stable for $\sim 10 \mathrm{sec}$, the operator froze it and stored the corresponding image in the machine. For each of these ROIs, the mean, minimum and maximum stiffness, as well as the standard deviation were automatically calculated, which increased with increasing tissue elasticity heterogeneity.

Histological evaluation. The samples of tumor tissue and retrieved lymph nodes were fixed in $10 \%$ formaldehyde solution, embedded in paraffin, cut into $4 \mathrm{~mm}$ sections and mounted on poly-lysine-coated slides. These tissue sections were stained using hematoxylin and eosin to confirm their histological diagnosis and other microscopic characteristics. Experienced pathologists were employed to ensure a high quality of pathological diagnosis.

\section{Results}

The patient had undergone total thyroidectomy and bilateral neck lymph node dissection, and an intraoperative 
pathological consultation to confirm the malignancy as lymph node metastasis. The total thyroid glands and bilateral neck lymph nodes were submitted for a pathological examination. The left lobe and right lobe measured $6 \times 4 \times 3.5$ and $5 \times 3 \times 2 \mathrm{~cm}^{3}$, respectively. Each lobe of thyroid was tan-to-white in color and lacking any nodular masses. Pathological diagnosis was DSVPTC in each lobe (Fig. 1A), with lymph node metastases in the bilateral neck.

A serological analysis revealed the following: Free T4, $0.70 \mathrm{ng} / \mathrm{dl}$, free T3,3.24 pg/ml, T3, $1.04 \mathrm{ng} / \mathrm{ml}, \mathrm{T} 4,8.14 \mu \mathrm{g} / \mathrm{dl}$ and TSH, $7.79 \mathrm{IU} / \mathrm{ml}$. Each antithyroid antibody was positive: Anti-thyroglobulin, >2,380.0 IU/ml and anti-thyroid peroxidase, $254.80 \mathrm{IU} / \mathrm{ml}$.

Thyroid ultrasonography revealed diffuse enlargement of the both lobes, heterogenous echogenicity without mass formation, diffuse scattered microcalcifications (Fig. 1B) and poor vascularization. It also revealed enlarged bilateral neck lymph nodes, an absence of a normal lymph hilum and structural disorder of the corticomedullary, without calcification.

SWE revealed stiff values of the thyroid: The mean stiffness was $99.7 \mathrm{kpa}$, the minimum stiffness was $59.1 \mathrm{kpa}$ and the maximum stiffness was $180.1 \mathrm{kpa}$ (Fig. 1C).

\section{Discussion}

Compared with classic PTC, DSVPTC occurs most frequently in young females and may be mistaken clinically for Hashimoto's thyroiditis $(3,7,9,12)$. It generally has a greater tendency for lymph node metastasis and distant metastasis, and worse disease-free survival compared with other PTC variants (8-11). Due to its strong aggressiveness and early metastasis, total thyroidectomy with lymph node dissection and postoperative radioiodine ablation are recommended, and a close follow-up is necessary $(3,7,9,10,12)$.

Although DSVPTC is rare (16), it is important for clinicians to recognize and consider the possibility of this disease, particularly because of its clinical resemblance to Hashimoto's thyroiditis $(3,9)$. Patients are frequently misdiagnosed and treated for Hashimoto's thyroiditis $(7,9,14)$. In the present case, the clinical presentation and serological findings were all indicative of Hashimoto's thyroiditis.

Sonography is generally considered as the most accurate imaging modality for the evaluation and characterization of thyroid nodules (13). However, the diffuse nature of DSVPTC often mimics chronic thyroiditis on US images and leads to treatment delay, particularly in the absence of a focal solid mass $(14,15)$. In the present case, thyroid ultrasonography revealed diffuse enlargement of each lobe and heterogenous echogenicity without focal solid mass formation. The preliminary ultrasonic diagnostic impression was of Hashimoto's thyroiditis. It is well known that microcalcifications within a solitary thyroid mass on sonography are generally regarded as the most reliable indicators of malignancy (17). Since the present case showed diffuse scattered microcalcifications, it is imperative to accurately identify the underlying papillary carcinoma. Subsequently, SWE were performed during the US examination.

Elastography is a sonographic method of assessing tissue stiffness. Numerous previous studies reported decreased elasticity of malignant thyroid nodules (18-20). Since the older variants of elastography determines tissue elasticity in relation to surrounding tissue (20), it may influence the diagnostic performance of diffuse disease, including DSVPTC, coexisting subacute thyroiditis and Hashimoto's thyroiditis. SWE is a novel, promising, user-independent and real-time, quantitative (20-24), but not widely available technique. In SWE (20-24), SWE is induced by a focused ultrasonic beam. Based on the received signals, the elasticity of the tissue is assessed in real-time and may be estimated both qualitatively and quantitatively. It is thought to be more objective, reliable and reproducible compared with older variants of elastography, as it does not require any compressive maneuvers $(20,22,24)$. Previous studies on SWE demonstrated very significant differences in elasticity between benign and malignant lesions $(20,21,23-25)$. To the best of our knowledge, SWE diagnosis of DSVPTC of the thyroid remains to be described.

Numerous previous studies of SWE have demonstrated that a cut-off value of maximum stiffness $>65 \mathrm{kPa}$ for diagnosing the malignant thyroid nodules had a sensitivity of $85.2 \%$ and specificity of $93.9 \%$ (24). The mean stiffness of Hashimoto's thyroiditis was $24.0 \pm 10.5 \mathrm{kpa}(21)$. In the present case, SWE showed stiff values of the thyroid: The mean stiffness was $99.7 \mathrm{kpa}$, the minimum stiffness was $59.1 \mathrm{kpa}$ and the maximum stiffness was $180.1 \mathrm{kpa}$. The maximum stiffness of the DSVPTC (180.1 kpa) was higher compared with the diagnostic criteria of malignant thyroid nodules $(65 \mathrm{kPa})$. This may suggest the benefit of diagnosing DSVPTC by SWE.

The present study was limited in terms of the study population. Since only 1 patient was assessed, the population was too small to draw any definite conclusions. Therefore, multi-center prospective studies with larger numbers of sample are required in future investigations.

In conclusion, SWE is an important imaging tool in the diagnosis of DSVPTC. Since patients with DSVPTC may present with typical clinicopathological features and initially appear to have Hashimoto's thyroiditis, a thorough clinical evaluation and an early diagnosis are important. DSVPTC requires more aggressive surgical intervention and it is therefore important to diagnose this as quickly as possible to ensure the correct treatment is provided.

\section{References}

1. Vickery AL Jr, Carcangiu ML, Johannessen JV and Sobrinho-Simoes M: Papillary carcinoma. Semin Diagn Pathol 2: 90-100, 1985.

2. Lam AK, Lo CY and Lam KS: Papillary carcinoma of thyroid: A 30-yr clinicopathological review of the histological variants. Endocr Pathol 16: 323-330, 2005.

3. Thompson LD, Wieneke JA and Heffess CS: Diffuse sclerosing variant of papillary thyroid carcinoma: A clinicopathologic and immunophenotypic analysis of 22 cases. Endocr Pathol 16: 331-348, 2005.

4. Chow SM, Chan JK, Law SC, Tang DL, Ho CM, Cheung WY, Wong IS and Lau WH: Diffuse sclerosing variant of papillary thyroid carcinoma-clinical features and outcome. Eur J Surg Oncol 29: 446-449, 2003.

5. Carcangiu ML and Bianchi S: Diffuse sclerosing variant of papillary thyroid carcinoma. Clinicopathologic study of 15 cases. Am J Surg Pathol 13: 1041-1049, 1989.

6. Imamura Y,Kasahara Y and Fukuda M: Multiple brain metastases from a diffuse sclerosing variant of papillary carcinoma of the thyroid. Endocr Pathol 11: 97-108, 2000. 
7. Akaishi J, Sugino K, Kameyama K, Masaki C, Matsuzu K, Suzuki A, Uruno T, Ohkuwa K, Shibuya H, Kitagawa W, et al: Clinicopathologic features and outcomes in patients with diffuse sclerosing variant of papillary thyroid carcinoma. World J Surg 39: 1728-1735, 2015.

8. Falvo L, Giacomelli L, D'Andrea V, Marzullo A, Guerriero G and de Antoni E: Prognostic importance of sclerosing variant in papillary thyroid carcinoma. Am Surg 72: 438-444, 2006.

9. Chen CC, Chen WC, Peng SL and Huang SM: Diffuse sclerosing variant of thyroid papillary carcinoma: Diagnostic challenges occur with Hashimoto's thyroiditis. J Formos Med Assoc 112: 358-362, 2013.

10. Fukushima M, Ito Y, Hirokawa M, Akasu H, Shimizu K and Miyauchi A: Clinicopathologic characteristics and prognosis of diffuse sclerosing variant of papillary thyroid carcinoma in Japan: An 18-year experience at a single institution. World J Surg 33: 958-962, 2009.

11. Regalbuto C, Malandrino P, Tumminia A, Le Moli R, Vigneri R and Pezzino V: A diffuse sclerosing variant of papillary thyroid carcinoma: Clinical and pathologic features and outcomes of 34 consecutive cases. Thyroid 21: 383-389, 2011.

12. Marshall CB: Diffuse sclerosing variant of papillary thyroid carcinoma preoperative diagnosis through imaging and cytology allows for optimal patient care. Pathology Case Reviews 20: $125-128,2015$

13. Pacini F, Schlumberger M, Dralle H, Elisei R, Smit JW and Wiersinga W; European Thyroid Cancer Taskforce: European consensus for the management of patients with differentiated thyroid carcinoma of the follicular epithelium. Eur J Endocrinol 154: 787-803, 2006.

14. Kim HS, Han BK, Shin JH, Ko EY, Sung CO, Oh YL and Song SY: Papillary thyroid carcinoma of a diffuse sclerosing variant: Ultrasonographic monitoring from a normal thyroid gland to mass formation. Korean J Radiol 11: 579-582, 2010.

15. Martín-Pérez E, Larrañaga E and Serrano P: Diffuse sclerosing variant of papillary carcinoma of the thyroid. Eur J Surg 164: 713-715, 1998 .

16. Sheu SY, Schwertheim S, Worm K, Grabellus F and Schmid KW: Diffuse sclerosing variant of papillary thyroid carcinoma: Lack of BRAF mutation but occurrence of RET/PTC rearrangements. Mod Pathol 20: 779-787, 2007.
17. Zhang Y, Xia D, Lin P, Gao L, Li G and Zhang W: Sonographic findings of the diffuse sclerosing variant of papillary carcinoma of the thyroid. J Ultrasound Med 29: 1223-1226, 2010.

18. Shuzhen C: Comparison analysis between conventional ultrasonography and ultrasound elastography of thyroid nodules. Eur J Radiol 81: 1806-1811, 2012.

19. Wang Y, Dan HJ, Dan HY, Li T and Hu B: Differential diagnosis of small single solid thyroid nodules using real-time ultrasound elastography. J Int Med Res 38: 466-472, 2010.

20. Liu BX, Xie XY, Liang JY, Zheng YL, Huang GL, Zhou LY, Wang $\mathrm{Z}, \mathrm{Xu} \mathrm{M}$ and Lu MD: Shear wave elastography versus real-time elastography on evaluation thyroid nodules: A preliminary study. Eur J Radiol 83: 1135-1143, 2014

21. Magri F, Chytiris S, Capelli V, Alessi S, Nalon E, Rotondi M, Cassibba S, Calliada F and Chiovato L: Shear wave elastography in the diagnosis of thyroid nodules: Feasibility in the case of coexistent chronic autoimmune Hashimoto's thyroiditis. Clin Endocrinol (Oxf) 76: 137-141, 2012.

22. Lee EJ, Jung HK, Ko KH, Lee JT and Yoon JH: Diagnostic performances of shear wave elastography: Which parameter to use in differential diagnosis of solid breast masses? Eur Radiol 23: 1803-1811, 2013.

23. Liu B, Liang J, Zheng Y, Xie X, Huang G, Zhou L, Wang W and $\mathrm{Lu} \mathrm{M}$ : Two-dimensional shear wave elastography as promising diagnostic tool for predicting malignant thyroid nodules: A prospective single-centre experience. Eur Radiol 25: 624-634, 2015.

24. Veyrieres JB, Albarel F, Lombard JV, Berbis J, Sebag F, Oliver C and Petit P: A threshold value in Shear Wave elastography to rule out malignant thyroid nodules: A reality? Eur J Radiol 81: 3965-3972, 2012.

25. Sebag F, Vaillant-Lombard J, Berbis J, Griset V, Henry JF, Petit P and Oliver C: Shear wave elastography: A new ultrasound imaging mode for the differential diagnosis of benign and malignant thyroid nodules. J Clin Endocrinol Metab 95: 5281-5288, 2010 